\title{
Controlling Phase Transitions in a 2D Model System
}

\author{
Modifying surface and boundary features lets researchers control how a \\ 2D model system transitions from a fluid to a crystalline phase.
}

\author{
By Erika K. Carlson
}

$\square$ hase transitions in 2D systems are of interest for a variety of research applications, but much about them remains mysterious. For example, researchers have been trying to figure out how two-step phase transitions seen in some 2D model systems (in which the fluid-to-crystalline transitions occur via an intermediate state) are affected when these models incorporate certain real-world features. Now, James Downs of the University of Nottingham, UK, and colleagues have investigated how phase transitions in their experimental model system depend on the textures of the surface it sits on and the boundaries that surround it [1].

The researchers nearly filled a flat hexagonal plate with a layer of spherical particles. They vibrated the plate to make the particles jitter around like molecules in a fluid. Then they slowed the vibration to mimic the cooling of that fluid, leading the particles to fall into ordered, crystalline arrangements.

Conducting this experiment on a smooth plate led to a two-step phase transition, with the particles passing through an

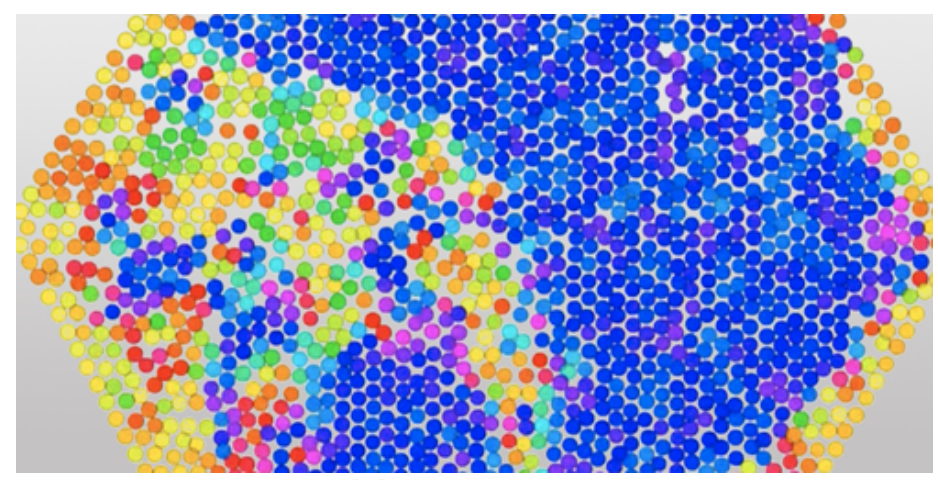

Credit: J. G. Downs et al. [1]

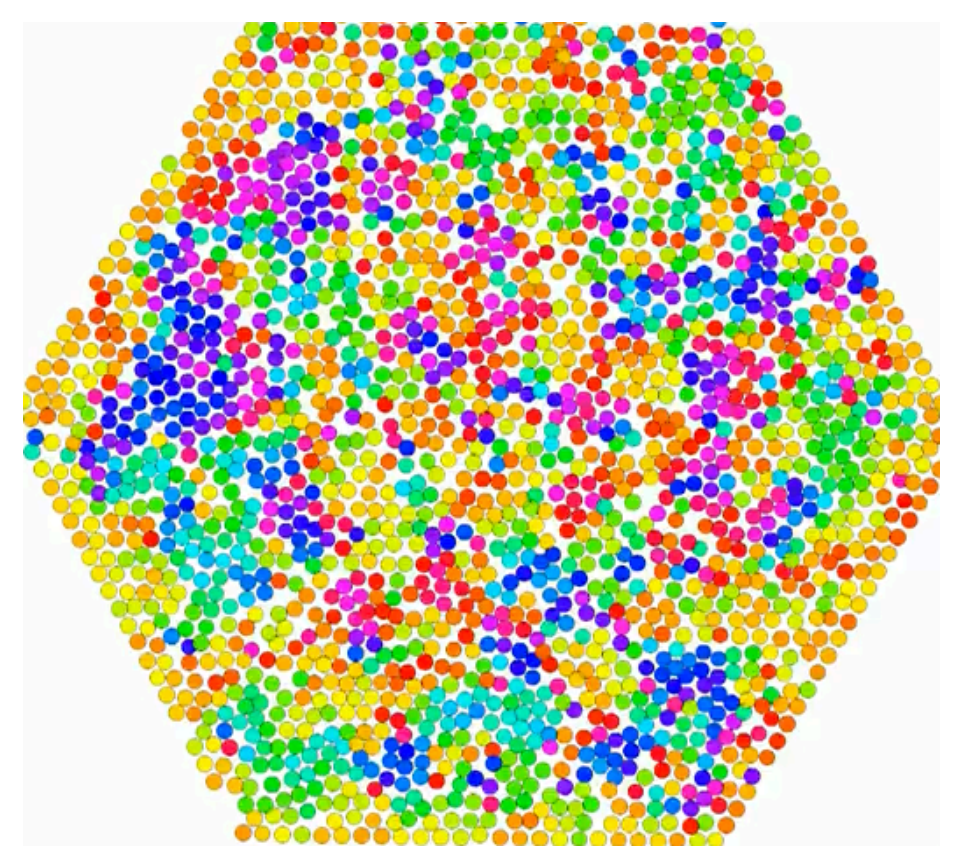

Video 1: This animation of the team's experimental data shows an ordered, crystalline structure (the growing mass of blue particles) arising in the model system of vibrating spherical particles as it "cools," i.e., as the particles' vibrations slow.

Credit: J. G. Downs et al. [1]

intermediate liquid-crystal-like "hexatic" phase. However, when the researchers used a plate with a periodic pattern of dimples on its surface, the particles settled directly into the crystalline state. The researchers also found that the structure of the hexagonal plate's boundaries could manipulate where the fluid and crystalline phases manifested when the system cooled through the phase transition. By adding certain textures, they could create "orderphobic" boundaries that deterred 
nearby particles from settling into a crystalline state and "orderphilic" boundaries that encouraged it. Further work using this or similar model systems could, for example, lend insight into how phase transitions in lipid membranes could affect the clustering of proteins in cell membranes.

Erika K. Carlson is a Corresponding Editor for Physics based in New
York City.

\section{REFERENCES}

1. J. G. Downs et al., "Topographic control of order in quasi-2D granular phase transitions,” Phys. Rev. Lett. 127, 268002 (2021). 